\title{
Concomitant Medication Start Relative to Reference Timepoint
}

National Cancer Institute

\section{Source}

National Cancer Institute. Concomitant Medication Start Relative to Reference

Timepoint. NCl Thesaurus. Code C87864.

An indication or description of the start of concomitant medication usage in relation to a specific point in time. 